\title{
Ultrametric Corona Problem and spherically complete fields
}

\author{
by Alain Escassut
}

\begin{abstract}
Let $K$ be a complete ultrametric algebraically closed field and let $A$ be the Banach $K$-algebra of bounded analytic functions in the "open" unit disk $D$ of $K$ provided with the Gauss norm. Let $\operatorname{Mult}(A, \|$. $\|)$ be the set of continuous multiplicative seminorms of $A$ provided with the topology of simple convergence, let $\operatorname{Mult}_{m}(A, \|$. \|) be the subset of the $\phi \in \operatorname{Mult}\left(A, \|\right.$. \|) whose kernel is a maximal ideal and let $\operatorname{Mult}_{a}(A, \|$. $\|)$ be the subset of the $\phi \in \operatorname{Mult}(A, \|$. \|) whose kernel is a maximal ideal of the form $(x-a) A$ with $a \in D$. We complete the characterization of continuous multiplicative norms of $A$ by proving that the Gauss norm defined on polynomials has a unique continuation to $A$ as a norm: the Gauss norm again. But we find prime closed ideals that are neither maximal nor null. The Corona problem on $A$ lies in two questions: is $\operatorname{Mult}_{a}(A, \|$. $\|)$ dense in $\operatorname{Mult}_{m}(A,\|\|$.$) ? Is it dense in \operatorname{Mult}(A,\|\|$.$) ? In a previous paper, Mainetti and$ Escassut showed if each maximal ideal of $A$ is the kernel of a unique $\phi \in \operatorname{Mult}_{m}(A,\|\cdot\|)$, then the answer to the first question is yes (which is the case when $K$ is strongly valued). Here we prove that this is also true when $K$ is spherically complete.
\end{abstract}

2000 Mathematics subject classification: Primary 12J25 Secondary 46S10

\section{Introduction and results.}

Let $B=H^{\infty}(D)$ be the unital Banach algebra of bounded analytic functions on the open unit disk $D$ in the complex plane. Each $a \in D$ defines a multiplicative linear functional $\phi_{a}$ on $B$ by "point evaluation" i.e. $\phi_{a}(f)=f(a)$. If a function $f$ lies in the kernel of all the $\phi_{a}$ then clearly $f=0$. This tells us that the set of all the $\phi_{a}$ is dense in the set $\Xi(B)$ of all non-zero multiplicative linear functionals on $B$ in the hull-kernel topology which is lifted from the kernels of the functionals, which are the maximal ideals of $B$ : each maximal ideal being of codimension 1 , it is the kernel of such a multiplicative functional.

The Corona Conjecture of Kakutani was that one also has density with respect to the weak topology (the topology of simple convergence on $\Xi(B)$ ). This was famously proved by Carleson in 1962 [4]. The key fact is that if $f_{1}, \ldots, f_{n}$ belong to $B$ and if there exists $d>0$ such that, for all $a \in D$ we have

$$
\left|f_{1}(z)\right|+\ldots \ldots+\left|f_{n}(z)\right|>d
$$

then the ideal generated by the $f_{1}, \ldots, f_{n}$ is the whole of $B$. People often transfer the name "Corona Statement" to this key fact. Actually, this Corona Statement implies that the Corona Conjecture is true, thanks to the fact that all maximal ideals of a $\mathbb{C}$-Banach algebra are of codimension 1 .

Now consider the situation in the non-archimedean context. Let $K$ be an algebraically closed field complete with respect to an ultrametric absolute value $\mid$. $\mid$. Given $a \in K$ 
and $r>0$, we denote by $d(a, r)$ the disk $\{x \in K|| x-a \mid \leq r\}$, by $d\left(a, r^{-}\right)$the disk $\{x \in K|| x-a \mid<r\}$, by $C(a, r)$ the circle $\{x \in K|| x-a \mid=r\}$ and set $D=d\left(0,1^{-}\right)$. Let $A$ be the $K$-algebra of bounded power series converging in $D$ which is complete with respect to the Gauss norm defined as $\left\|\sum_{n=1}^{\infty} a_{n} x^{n}\right\|=\sup _{n \in \mathbb{N}}\left|a_{n}\right|$ : we know that this norm actually is the norm of uniform convergence on $D[8],[15]$.

In [19] the Corona problem was considered in a similar way as it is on the field $\mathbb{C}$ [4], [14]: the author asked the question whether the set of maximal ideals of $A$ defined by the points of $D$ (which are well known to be of the form $(x-a) A$ ) is dense in the whole set of maximal ideals with respect to a so-called "Gelfand Topology". In fact, as explained in [10], this makes no sense because the maximal ideals which are not of the form $(x-a) A$ are of infinite codimension [10]. Consequently, a Corona problem should be defined in a different way, as explained in [10]. However, in [19] a "Corona Statement" similar to this mentioned above was shown in our algebra $A$ and it is useful in the present paper as it was in [10].

First, here we will complete the characterization of continuous multiplicative norms on $A$ (begun in [10]). Next, we will look for prime closed ideals other than maximal ideals and the zero ideal: we'll show such ideals do exist. And finally, when the field is spherically complete, we will show that each maximal ideal is the kernel of only one continuous multiplicative semi-norm, which implies the density of the set of continuous multiplicative semi-norms whose kernel is a maximal ideal of codimension 1 in the set of all of continuous multiplicative semi-norms whose kernel is a maximal ideal ( it is well known that the field $\mathbb{C}_{p}$ is not spherically complete [17] but it admits a spherical completion). The main tool to solve this problem is the ultrametric holomorphic functional calculus [7], [9], but we also have to examine ultrafilters.

Given a commutative $K$-algebra $B$ with unity, provided with a $K$-algebra norm $\|$. $\|$, the set of continuous multiplicative $K$-algebra semi-norms of $B$ was studied in many works [13], [7], [8], [9], [1] and is usually denoted by $\operatorname{Mult}(B, \|$. \|) [13], [7], [8], [9]. For each $\phi \in \operatorname{Mult}(B,\|\|$.$) , we denote by \operatorname{Ker}(\phi)$ the closed prime ideal of the $f \in B$ such that $\phi(f)=0$. The set of the $\phi \in \operatorname{Mult}(B,\|\|$.$) such that \operatorname{Ker}(\phi)$ is a maximal ideal is denoted by $\operatorname{Mult}_{m}(B,\|\|$.$) , the set of the \phi \in \operatorname{Mult}(B,\|\|$.$) such that \operatorname{Ker}(\phi)$ is a maximal ideal of codimension 1 is denoted by $\operatorname{Mult}_{a}(B,\|\|$.$) and here, the set of continuous multiplicative$ norms of $A$ will be denoted by $\operatorname{Mult}_{o}(B, \|$. \|).

We know that $\sup \{\phi(f) \mid \phi \in \operatorname{Mult}(B,\|\|)\}=.\lim _{n \rightarrow \infty}\left(\left\|f^{n}\right\|\right)^{\frac{1}{n}} \forall f \in B$ [13], [9]. On the other hand, $\operatorname{Mult}(B,\|\|$.$) is provided with the topology of simple convergence and is$ compact for this topology.

We know that for every $\mathcal{M} \in \operatorname{Max}(B)$, there exists at least one $\phi \in \operatorname{Mult}_{m}(B, \|$. $\|)$ such that $\operatorname{Ker}(\phi)=\mathcal{M}$ but in certain cases, there exist infinitely many $\phi \in \operatorname{Mult}_{m}(B,\|\|$. such that $\operatorname{Ker}(\phi)=\mathcal{M}$ [6], [9]. A maximal ideal $\mathcal{M}$ of $B$ is said to be univalent if there is only one $\phi \in \operatorname{Mult}_{m}(B, \|$. $\|)$ such that $\operatorname{Ker}(\phi)=\mathcal{M}$ and the algebra $B$ is said to be multbijective if every maximal ideal is univalent (so, non-multbijective commutative Banach $K$-algebras with unity do exist). 
Thus, the ultrametric Corona problem may be viewed at two levels: 1) Is $\operatorname{Mult}_{a}(A,\|\|$.$) dense in \operatorname{Mult}_{m}(A, \|$. $\|$ ) (with respect to the topology of simple convergence)?

2) Is $\operatorname{Mult}_{a}(A,\|\|$.$) dense in \operatorname{Mult}(A,\|\|$.$) (with respect to the same topology )?$

Actually, this way to set the Corona problem on an ultrametric field is not really different from the original problem once considered on $\mathbb{C}$ because on a commutative $\mathbb{C}$ Banach algebra with unity, all continuous multiplicative semi-norms are known to be of the form $|\chi|$ where $\chi$ is a character of $A$. Thus the Corona problem was equivalent to show that the set of multiplicative semi-norms defined by the points of the open disk was dense inside the whole set of continuous multiplicative semi-norms, with respect to the topology of simple convergence.

Let $K$ be an algebraically closed field complete with respect to an ultrametric absolute value $|$.$| . Given a \in K$ and $r>0$, we denote by $d(a, r)$ the disk $\{x \in K|| x-a \mid \leq r\}$, by $d\left(a, r^{-}\right)$the disk $\{x \in K|| x-a \mid<r\}$, by $C(a, r)$ the circle $\{x \in K|| x-a \mid=r\}$ and set $D=d\left(0,1^{-}\right)$. Let $A$ be the $K$-algebra of bounded power series converging in $D$ which is complete with respect to the Gauss norm defined as $\left\|\sum_{n=1}^{\infty} a_{n} x^{n}\right\|=\sup _{n \in \mathbb{N}}\left|a_{n}\right|$ : we know that this norm actually is the norm of uniform convergence on $D$ [8], [15].

In [19] the Corona problem was considered in a similar way as it is on the field $\mathbb{C}$ [14]: the author asked the question whether the set of maximal ideals of $A$ defined by the points of $D$ (which are well known to be of the form $(x-a) A$ ) is dense in the whole set of maximal ideals with respect to a so-called "Gelfand Topology". In fact, as explained in [10], this makes no sense because the maximal ideals which are not of the form $(x-a) A$ are of infinite codimension [10]. Consequently, a Corona problem should be defined in a different way, as explained in [10].

First, we mean to complete the characterization of multiplicative norms on $A$ (begun in [10]). On the other hand we want to show the density of the set of continuous multiplicative semi-norms whose kernel is a maximal ideal of codimension 1 in the set of all of continuous multiplicative semi-norms whose kernel is a maximal ideal. However, for this last question, we need to take for $K$ a spherically complete field (it is well known that $\mathbb{C}_{p}$ is not spherically complete [17] .

Given a commutative $K$-algebra $B$ with unity, provided with a $K$-algebra norm $\|$. $\|$, the set of continuous multiplicative $K$-algebra semi-norms of $B$ was studied in many works [13], [7], [8], [9], [1] and is usually denoted by $\operatorname{Mult}(B, \|$. \|) [13], [7], [8], [9]. For each $\phi \in \operatorname{Mult}(B, \|$. $\|)$, we denote by $\operatorname{Ker}(\phi)$ the closed prime ideal of the $f \in B$ such that $\phi(f)=0$. The set of the $\phi \in \operatorname{Mult}(B,\|\|$.$) such that \operatorname{Ker}(\phi)$ is a maximal ideal is denoted by $\operatorname{Mult}_{m}(B,\|\cdot\|)$, the set of the $\phi \in \operatorname{Mult}(B,\|\cdot\|)$ such that $\operatorname{Ker}(\phi)$ is a maximal ideal of codimension 1 is denoted by $\operatorname{Mult}_{a}(B,\|\|$.$) and here, the set of continuous multiplicative$ norms of $A$ will be denoted by $\operatorname{Mult}_{o}(B, \|$. $\|)$.

We know that $\sup \{\phi(f) \mid \phi \in \operatorname{Mult}(B,\|\cdot\|)\}=\lim _{n \rightarrow \infty}\left(\left\|f^{n}\right\|\right)^{\frac{1}{n}} \forall f \in B$ [13], [9]. On the other hand, $\operatorname{Mult}(B,\|\|$.$) is provided with the topology of simple convergence and is$ compact for this topology. 
We know that for every $\mathcal{M} \in \operatorname{Max}(B)$, there exists at least one $\phi \in \operatorname{Mult}_{m}(B, \|$. $\|)$ such that $\operatorname{Ker}(\phi)=\mathcal{M}$ but in certain cases, there exist infinitely many $\phi \in \operatorname{Mult}_{m}(B,\|\|$. such that $\operatorname{Ker}(\phi)=\mathcal{M}$ [6], [9]. A maximal ideal $\mathcal{M}$ of $B$ is said to be univalent if there is only one $\phi \in \operatorname{Mult}_{m}(B, \|$. \|) such that $\operatorname{Ker}(\phi)=\mathcal{M}$ and the algebra $B$ is said to be multbijective if every maximal ideal is univalent (so, non-multbijective commutative Banach $K$-algebras with unity do exist).

Thus, the ultrametric Corona problem may be viewed at two levels:

1) Is $\operatorname{Mult}_{a}(A,\|\|$.$) dense in \operatorname{Mult}_{m}(A, \|$. $\|$ ) (with respect to the topology of simple convergence)?

2) Is $\operatorname{Mult}_{a}(A,\|\|$.$) dense in \operatorname{Mult}(A,\|\|$.$) (with respect to the same topology )?$

Actually, this way to set the Corona problem on an ultrametric field is not really different from the original problem once considered on $\mathbb{C}$ because on a commutative $\mathbb{C}$ Banach algebra with unity, all continuous multiplicative semi-norms are known to be of the form $|\chi|$ where $\chi$ is a character of $A$. Thus the Corona problem was equivalent to show that the set of multiplicative semi-norms defined by the points of the open disk was dense inside the whole set of continuous multiplicative semi-norms, with respect to the topology of simple convergence.

Remark: Given a filter $\mathcal{G}$, if for every $f \in A,|f(x)|$ admits a limit $\varphi_{\mathcal{G}}(f)$ along $\mathcal{G}$, the function $\varphi_{\mathcal{G}}$ obviously belongs to $\operatorname{Mult}(A,\|\cdot\|)$. Moreover, it clearly lies in the closure of $\operatorname{Mult}_{a}(A,\|\cdot\|)$. Consequently, if we can prove that every element of $\operatorname{Mult}_{m}(A, \|$. $\|)$ is of the form $\varphi_{\mathcal{G}}$, with $\mathcal{G}$ a certain filter on $D$, Question 1) is solved. And similarly, if we could prove that every element of $\operatorname{Mult}(A,\|\|$.$) is of the form \varphi_{\mathcal{G}}$, Question 2) would be solved.

Studying such problems first requires to know the nature of continuous multiplicative semi-norms on $A$. Here the first part of this study consists of studying continuous multiplicative norms on $A$ by completing a study made in [10]. We have to recall the role of circular filters and this of ultrafilters.

Definitions and notation: Let $a \in D$ and let $R \in] 0,1]$. Given $r, s \in \mathbb{R}$ such that $0<r<s$ we set $\Gamma(a, r, s)=\{x \in K|r<| x-a \mid<s\}$.

We call circular filter of center $a$ and diameter $R$ on $D$ the filter $\mathcal{F}$ which admits as a generating system the family of sets $\Gamma\left(\alpha, r^{\prime}, r^{\prime \prime}\right) \cap D$ with $\alpha \in d(a, R), r^{\prime}<R<r^{\prime \prime}$, i.e. $\mathcal{F}$ is the filter which admits for basis the family of sets of the form $D \cap\left(\bigcap_{i=1}^{q} \Gamma\left(\alpha_{i}, r_{i}^{\prime}, r_{i}^{\prime \prime}\right)\right)$ with $\alpha_{i} \in d(a, R), r_{i}^{\prime}<R<r_{i}^{\prime \prime} \quad(1 \leq i \leq q, q \in \mathbb{N})$.

Recall that the field $K$ is said to be spherically complete if every decreasing sequence of disks has a non-empty intersection (it is known that $\mathbb{C}_{p}$ is not spherically complete but it has a spherical completion).

In a field which is not spherically complete, one has to consider decreasing sequences of disks $\left(D_{n}\right)$ with an empty intersection. We call circular filter with no center, of canonical 
basis $\left(D_{n}\right)$ the filter admitting for basis the sequence $\left(D_{n}\right)$ and the number $\lim _{n \rightarrow \infty} \operatorname{diam}\left(D_{n}\right)$ is called diameter of the filter.

Finally the filter of neighborhoods of a point $a \in D$ is called circular filter of the neighbourhoods of $a$ on $D$ and its diameter is 0 . Given a circular filter $\mathcal{F}$, its diameter is denoted by $\operatorname{diam}(\mathcal{F})$.

Here, we shall denote by $\mathcal{W}$ the circular filter on $D$ of center 0 and diameter 1 and by $\mathcal{Y}$ the filter admitting for basis the family of sets of the form $\Gamma(0, r, 1) \backslash\left(\bigcup_{n=0}^{\infty} d\left(a_{n}, r_{n}^{-}\right)\right)$ with $a_{n} \in D, r_{n} \leq\left|a_{n}\right|$ and $\lim _{n \rightarrow \infty}\left|a_{n}\right|=1$.

An ultrafilter $\mathcal{U}$ on $D$ will be called coroner ultrafilter if it is thinner than $\mathcal{W}$. Similarly, a sequence $\left(a_{n}\right)$ on $D$ will be called a coroner sequence if its filter is a coroner filter, i.e. if $\lim _{n \rightarrow+\infty}\left|a_{n}\right|=1$.

Let $\psi \in \operatorname{Mult}(A,\|\|$.$) . Then \psi$ will be said to be coroner if its restriction to $K[x]$ equal to $\|$.$\| .$

In [10] regular ultrafilters were defined: Let $\left(a_{n}\right)_{n \in \mathbb{N}}$ be a coroner sequence in $D$. The sequence is called a regular sequence if $\inf _{j \in \mathbb{N}} \prod_{\substack{n \in \mathbb{N} \\ n \neq j}}\left|a_{n}-a_{j}\right|>0$.

An ultrafilter $\mathcal{U}$ is said to be regular if it is thinner than a regular sequence. Thus, by definition, a regular ultrafilter is a coroner ultrafilter.

Two coroner ultrafilters $\mathcal{F}, \mathcal{G}$ are said to be contiguous if for every subsets $F \in \mathcal{F}, G \in$ $\mathcal{G}$ of $D$ the distance from $F$ to $G$ is null.

On $K[x]$, circular filters on $K$ are known to characterize multiplicative semi-norms by associating to each circular filter $\mathcal{F}$ the multiplicative semi-norm $\varphi_{\mathcal{F}}$ defined as $\varphi_{\mathcal{F}}(f)=$ $\lim _{\mathcal{F}}|f(x)|[12],[7],[8],[9]$.

We know that every $f \in A$ is an analytic element in each disk $d(a, r)$ whenever $r \in] 0,1[$ [8]. Consequently, by classical results [8], several properties of polynomials have continuation to $A$ : given a circular filter $\mathcal{F}$ on $D$ of diameter $<1$, for every $f \in A,|f(x)|$ has a limit along $\mathcal{F}$ denoted by $\varphi_{\mathcal{F}}(f)$ and then $\varphi_{\mathcal{F}}$ is a continuous multiplicative seminorm on $A$. In particular, given $a \in D$ and $r \in] 0,1$, if we consider the circular filter $\mathcal{F}$ of center $a$ and diameter $r$, we denote by $\varphi_{a, r}$ the multiplicative semi-norm $\varphi_{\mathcal{F}}$ which actually is defined by $\varphi_{a, r}(f)=\lim _{|x-a| \rightarrow r}|f(x)|$ and is a norm whenever $\operatorname{diam}(\mathcal{F})>0$. So, if $\mathcal{F}$ is the circular filter of center 0 and diameter $r$, we set $|f|(r)=\varphi_{\mathcal{F}}(f)$. Next, $\varphi_{\mathcal{W}}$ defines the Gauss norm on $K[x]$ and therefore admits a natural continuation to $A$ as $\left\|\sum_{n=1}^{\infty} a_{n} x^{n}\right\|=\sup _{n \in \mathbb{N}}\left|a_{n}\right|$. However, by [10] we know that this continuation is far from unique.

So, the problem is first to determine whether such multiplicative semi-norms defined on $K[x]$ by circular filters on $D$ have a unique continuation to $A$.

Theorem A was proved in [10]:

Theorem A: Let $\mathcal{F}$ be a circular filter on $D$ of diameter $r<1$. Then $\varphi_{\mathcal{F}}$ admits a unique continuation to $A$ defined by $\varphi_{\mathcal{F}}(f)=\lim _{\mathcal{F}}|f(x)|$ and $\varphi_{\mathcal{F}}$ is a norm on $A$. 
Thus, the question arising here is the continuation to $A$ of the Gauss norm defined on $K[x]$. The problem, then is not this simple: we have to consider coroner ultrafilters.

Every ultrafilter $\mathcal{U}$ on $D$ defines an element $\varphi_{\mathcal{U}}$ of $\operatorname{Mult}(A,\|\cdot\|)$ as $\varphi_{\mathcal{U}}(f)=\lim _{\mathcal{U}}|f(x)|$ : such a limit does exist because each function $f \in A$ is bounded and therefore $|f(x)|$ takes values in the compact $[0,\|f\|]$.

Recall now the following theorem (Corollary 12.1 in [10]):

Definitions: An element $f \in A$ is said to be quasi-invertible if it has finitely many zeroes. Then we know that such an element is of the form $P g$ with $P \in K[x], P$ having all its zeroes in $D$ and $g$ an invertible element of the algebra $A$.

Given a filter $\mathcal{F}$ on $U$ we denote by $\mathcal{J}(\mathcal{F})$ the ideal of the $f \in A$ such that $\lim _{\mathcal{F}}(f)=0$.

A maximal ideal $\mathcal{M}$ of $A$ will be said to be coroner (resp. regular) if there exists a coroner (resp. regular) ultrafilter $\mathcal{U}$ such that $\mathcal{M}=\mathcal{J}(\mathcal{U})$.

Theorem B: Let $\mathcal{M}$ be a maximal ideal of $A$. Either $\mathcal{M}$ is of codimension 1 and then it is of the form $(x-a) A(a \in D)$, or it is of infinite codimension and then it is coroner. Moreover, if $\mathcal{M}$ be of infinite codimension, then:

i) $\varphi_{\mathcal{U}}$ belongs to the closure of $\operatorname{Mult}_{a}(A,\|\|$.$) .$

ii) Given any $f \in \mathcal{M}, f$ is not quasi-invertible.

By Theorem 23 [10], we have this:

Theorem C: Let $\mathcal{U}$ be a regular ultrafilter. Then $\mathcal{J}(\mathcal{U})$ is a regular maximal ideal of $A$.

Remark: Characterizing the coroner ultrafilters $\mathcal{U}$ such that $\mathcal{J}(\mathcal{U})$ is a maximal ideal appears very hard. For instance, consider an ultrafilter $\mathcal{U}$ thinner than $\mathcal{Y}$. It is a coroner ultrafilter. But $\mathcal{J}(\mathcal{U})=\{0\}$. Indeed, suppose a non-identically zero function $f$ lies in $\mathcal{J}(\mathcal{U})$. Let $\left(a_{n}\right)$ be its sequence of zeroes, set $r_{n}=\left|a_{n}\right|, n \in \mathbb{N}$, and let $E=D \backslash \bigcup_{n=0}^{\infty} d\left(a_{n}, r_{n}^{-}\right)$. Clearly $|f(x)|=|f|(|x|) \forall x \in E$. However, $E$ belongs to $\mathcal{Y}$ and therefore, $\mathcal{U}$ is secant with $E$, a contradiction with the hypothesis $f \in \mathcal{J}(\mathcal{U})$.

On the other hand, the mapping $\mathcal{J}$ from the set of coroner ultrafilters to the set of ideals of $A$ is not injective: as noticed in [10], two contiguous coroner ultrafilters define the same ideal.

Now, two corollaries derive from Theorems B and C:

Corollary C1: If $A$ is multbijective, then for every $\phi \in \operatorname{Mult}_{m}(A, \|$. $\|)$ there exists a coroner ultrafilter $\mathcal{U}$ such that $\phi=\varphi_{\mathcal{U}}$.

Corollary C2: If $A$ is multbijective, then $\operatorname{Mult}_{a}(A,\|\|$.$) is dense in \operatorname{Mult}_{m}(A,\|\|$.$) .$

Corollaries $\mathrm{C} 1$ and $\mathrm{C} 2$ have immediate applications to the case when $K$ is strongly valued. 
Definitions and notation: The field $K$ is said to be strongly valued if at least its residue class field or its value group is not countable [8].

Theorem D: If $K$ is strongly valued, every commutative $K$-Banach algebra with unity is multbijective [7], [9].

Corollary D1: If $K$ is strongly valued, then for every $\phi \in \operatorname{Mult}_{m}(A, \|$. $\|)$ there exists a coroner ultrafilter $\mathcal{U}$ such that $\phi=\varphi_{\mathcal{U}}$. Moreover, Mult ${ }_{a}(A, \|$. $\|)$ is dense in $\operatorname{Mult}_{m}(A,\|\|$.$) .$

Thus, by Theorems $\mathrm{B}$ and $\mathrm{D}$, if an element $\psi \in \operatorname{Mult}(A,\|\|$.$) is neither the Gauss$ norm nor of the form $\varphi_{\mathcal{F}}$ on the whole set $A$, with $\mathcal{F}$ a circular filter on $D$ of diameter $r<1$, then, its restriction to $K[x]$ must be the Gauss norm on $K[x]$. So its kernel is a prime closed ideal included in a maximal ideal of the form $\mathcal{J}(\mathcal{U})$, with $\mathcal{U}$ a coroner ultrafilter.

Here we shall first examine the problem of the continuation of $\varphi_{\mathcal{W}}$ to $A$ through multiplicative norms, what was not done in [10].

Notation: Let $F$ be a field, let $R$ be a commutative $F$-algebra with unity and let $\mathcal{D}$ be a derivation on $R$. Let $J$ be an ideal of $R$. We will denote by $\widetilde{J}$ the set $\left\{f \in R \mid \mathcal{D}^{(n)} \in\right.$ $J \forall n \in \mathbb{N}\}$.

On $A$ we shall apply this notation to the usual derivation of functions. Let $\psi \in$ $\operatorname{Mult}(A, \|$. \|). Here we set $\operatorname{Subker}(\psi)=\widetilde{\operatorname{Ker}(\psi)}$.

In [10], we asked the question whether there exist prime closed ideals which are neither zero nor maximal ideals. We are now able to answer this question, by noticing first a quite easy theorem whose proof mainly holds in basic algebraic considerations:

Theorem 1: Let $F$ be a field, let $R$ be a commutative $F$-algebra with unity and let $\mathcal{D}$ be $a$ derivation on $R$. Let $J$ be an ideal of $R$. Then $\widetilde{J}$ is an ideal of $R$ and $\widetilde{\widetilde{J}})=\widetilde{J}$. Moreover, if $F$ is of characteristic 0 and if $J$ is prime, so is $\widetilde{J}$.

Since $\left\|f^{\prime}\right\| \leq\|f\| \forall f \in A$, we can derive Corollary 1.1:

Corollary 1.1: Suppose $K$ is of characteristic zero. Let $\mathcal{P}$ be a prime ideal of $A$. Then $\widetilde{\mathcal{P}}$ is a prime ideal of $A$ such that $\widetilde{(\widetilde{\mathcal{P}})}=\widetilde{\mathcal{P}}$. Moreover, if $\mathcal{P}$ is closed, so is $\widetilde{\mathcal{P}}$.

Corollary 1.2: Suppose $K$ is of characteristic zero. Let $\psi \in \operatorname{Mult}(A, \|$. $\|)$. Then Subker $(\psi)$ is a prime closed ideal.

In order to prove Theorem 3 and give counterexample to Theorem 1 when $K$ is of characteristic $p \neq 0$, we shall state Theorem 2:

Theorem 2: $\quad$ There exist regular maximal ideals $\mathcal{M}$ of $A$ and $f \in \mathcal{M}$, having a sequence of zeroes of order 1 and no other zeroes, such that $f^{\prime} \notin \mathcal{M}$, and such that $\widetilde{M} \neq\{0\}$. 
Theorem 3: $\quad$ Suppose $K$ is spherically complete and let $\mathcal{M}$ be a regular maximal ideal of A. There exists $f \in \mathcal{M}$, having a sequence of zeroes of order 1 and no other zeroes, such that $f^{\prime} \notin \mathcal{M}$.

Remarks: 1) Now we may notice that when the field is of characteristic 2 , it is easy to show that for certain maximal ideals $\mathcal{M}$ of $A, \widetilde{\mathcal{M}}$ is not prime. Indeed, by Theorem 2 , there exists a regular maximal ideal $\mathcal{M}$ and $f \in \mathcal{M}$ such that $f^{\prime} \notin \mathcal{M}$. Hence $f$ does not belong to $\widetilde{\mathcal{M}}$. Now consider $g=f^{2}$. Then $g^{\prime}=2 f f^{\prime}=0$ hence $g^{(n)} \in \mathcal{M} \forall n \in \mathbb{N}$. If $K$ is of characteristic 3 , we can also construct a similar but less simple counter-example.

2) In the algebra of bounded complex holomorphic functions in the open unit disk of $\mathbb{C}$, the derivation is not an endomorphism. Consequently, ideals of the form $\widetilde{P}$ do not exist.

Following Theorem A,we can now complete the characterization of continuous multiplicative norms on $A$.

Theorem 4: Let $\psi \in \operatorname{Mult}(A,\|\|$.$) be coroner. Then Subker (\psi)$ is not null. Moreover, if $K$ is spherically complete, then, for every $f \in A$ such that $\psi(f)<\|f\|$, there exists $g \in \operatorname{Subker}(\psi)$ admitting no zero but zeroes of $f$ and admitting each zero of $f$ as a zero of order superior or equal to its order as a zero of $f$.

Corollary 4.1: Let $\psi \in \operatorname{Mult}(A,\|\|$.$) be coroner. Then \psi$ is not a norm.

By Theorem A and Theorem 1, we now can state Corollary 4.1:

Corollary 4.2: Let $\psi \in \operatorname{Mult}(A, \|$. $\|)$ be a norm. If $\psi$ is not $\|$. $\|$, there exists a circular filter $\mathcal{F}$ on $D$, of diameter $r<1$, such that $\psi=\varphi_{\mathcal{F}}$.

On the other hand, each coroner maximal ideal is the kernel of some coroner continuous multiplicative semi-norm of $A$. Consequently:

Corollary 4.3: Let $\mathcal{M}$ be a coroner maximal ideal of $A$. Then $\widetilde{\mathcal{M}}$ is not null.

Concerning the Corona Problem, we may notice this:

Corollary 4.4: $\operatorname{Mult}_{o}(A,\|\|$.$) is included in the closure of \operatorname{Mult}_{a}(A,\|\|$.$) .$

By Theorems 3 and 4, we can derive Theorem 5:

Theorem 5: $\quad$ Let $K$ be spherically complete and $\mathcal{M}$ be a regular maximal ideal. Then $\widetilde{\mathcal{M}}$ is neither null nor equal to $\mathcal{M}$.

Remark: The prime closed ideal we shall construct, in the proof of Theorem 5, which is neither null nor maximal, does not seem to be the kernel of an element of $\operatorname{Mult}(A, \|$. $\|)$. Recall that in [2] an example of Banach-K-algebra of analytic elements with no divisors of zero, admitting no continuous multiplicative norm, was constructed.

Corollary 5.1: Suppose $K$ is of characteristic zero. Then $A$ admits prime closed ideals that are neither null nor maximal ideals. Moreover, if $K$ is spherically complete, then 
every regular maximal ideal $\mathcal{M}$ of $A$ contains a prime closed ideal $\widetilde{\mathcal{M}}$ that is neither null nor equal to $\mathcal{M}$.

Next, we will consider continuous multiplicative semi-norms whose kernels are maximal ideals when the field is spherically complete and we can answer the first question asked at the beginning:

Theorem 6: If $K$ is spherically complete, then $A$ is multbijective.

Corollary 6.1: If $K$ is spherically complete, then for every $\phi \in \operatorname{Mult}_{m}(A, \|$. $\|) \backslash$ $\operatorname{Mult}_{a}\left(A, \|\right.$. \|) there exists a coroner ultrafilter $\mathcal{U}$ such that $\phi=\varphi_{\mathcal{U}}$.

Corollary 6.2: If $K$ is spherically complete, then $\operatorname{Mult}_{a}(A,\|\|$.$) is dense in M u l t_{m}(A,\|\|$.$) .$

In [10] we considered the following conjectures:

$A$ is multbijective no matter what the complete algebraically closed field $K$.

This conjecture obviously implies that every $\phi \in \operatorname{Mult}_{m}(A,\|\|$.$) is of the form \varphi_{\mathcal{U}}$ with $\mathcal{U}$ an ultrafilter on $D$, and hence that $\operatorname{Mult}_{a}(A, \|$. $\|)$ is dense in $\operatorname{Mult}_{m}(A, \|$. $\|)$, whatever the complete algebraically closed field $K$. It seems very unikely that $A$ might be non-multbijective with certain field which is not spherically complete such as $\mathbb{C}_{p}$. However, in Proposition 13 below, due to Lazard's problem [16], the hypothesis " $K$ is spherically complete" is crucial to factorize a function $h$ in the form $\widehat{h} \widetilde{h}$.

Is $\operatorname{Mult}_{a}(A,\|\cdot\|)$ is dense in $\operatorname{Mult}(A,\|\|$.$) ?$

This seems much more difficult. By Corollary 4.4 we prove that all continuous multiplicative norms are known and do belong to the closure of $\operatorname{Mult}_{a}(A, \|$. $\|)$. And by Corollary 6.1 , we have proved that (provided $K$ is spherically complete, or strongly valued), every element of $\operatorname{Mult}(A,\|\cdot\|)$ is defined by a limit of $|f(x)|$ on a filter, except maybe some elements whose kernel is a prime closed ideal that is neither zero nor maximal. Thus, if $\operatorname{Mult}_{a}(A,\|\cdot\|)$ were not dense in $\operatorname{Mult}(A,\|\cdot\|)$, this would only be due to such elements.

But on the other hand, by Theorem 2, we have seen that in characteristic zero there exist prime closed ideals that are neither null nor maximal ideals. Might they be kernels of continuous multiplicative semi-norms? A natural candidate for such a semi-norm admitting for kernel $\operatorname{Subker}(\psi)$ would be defined as $\widetilde{\psi}(f)=\sup _{n \in \mathbb{N}}\left(\psi\left(f^{(n)}\right)\right.$. But this semi-norm is not multiplicative when the residue characteristic of $K$ is $p \neq 0$. If $p=0$, the answer is not clear.

Acknowledgement: I am grateful to Marie-Claude Sarmant and Kamal Boussaf for many remarks and to the Editors and the referee for suggestions on the introduction.

\section{The Proofs.}


Proof of Theorem 1: $\widetilde{J}$ obviously is an ideal of $R$. For convenience, let us set $f^{(n)}=$ $\mathcal{D}^{(n)}(f), n \in \mathbb{N}$, with $f^{(0)}=f$. Now let $f \in \widetilde{J}$ and consider $f^{(k)}$. Given any $n \in \mathbb{N}$, we have $\left(f^{(k)}\right)^{(n)}=f^{(n+k)} \in J$, hence $f^{(k)}$ belongs to $\widetilde{J}$. Consequently, $f^{(k)}$ belongs to $\widetilde{J}$ for every $k \in \mathbb{N}$. So $\widetilde{J}$ is included in $\widetilde{(\widetilde{J})}$ and therefore is equal to this.

Now, suppose that $F$ is of characteristic 0 and that $J$ is prime. We will check that $\widetilde{J}$ is a prime ideal. Let $f, g \in R \backslash \widetilde{J}$. So there exist integers $k, l \in \mathbb{N}$ such that $f^{(j)} \in J \forall j<k, g^{(j)} \in J \forall j<l$ and $\left.f^{(k)} \notin J, g^{(l)}\right) \notin J$. Consider

$(f g)^{(k+l)}=\sum_{j=0}^{k+l}\left(\begin{array}{c}k+l \\ j\end{array}\right) f^{(j)} g^{(k+l-j)}$. It is easily seen that

$f^{(j)} g^{(k+l-j)} \in J \forall j<k$ and $\forall j>k$ and that $f^{(k)} g^{(l)} \notin J$ because $J$ is prime. Consequently, since $F$ is of characteristic $0, \sum_{j=0}^{k+l}(k+l c r j) f^{(j)} g^{(k+l-j)} \notin J$, which shows that $\widetilde{J}$ is prime.

In the proof of Theorems we shall need several basic results. Lemma 1 is immediate and Lemma 2 is well known [8]:

Lemma 1: Let $\sum_{n=0}^{\infty} u_{n}$ be a converging series with positive terms. There exists a sequence of strictly positive integers $t_{n} \in \mathbb{N}$ satisfying

$t_{n} \leq t_{n+1}, n \in \mathbb{N}$,

$\lim _{n \rightarrow \infty} t_{n}=+\infty$

$\sum_{m=0}^{\infty} t_{m} u_{m}<+\infty$.

Lemma 2: $\quad$ Let $f \in A$ be not quasi-invertible and let $\left(a_{n}\right)_{n \in \mathbb{N}}$ be the sequence of zeroes with respective multiplicity $q_{n}$. Then the series $\sum_{n=0}^{\infty} q_{n} \log \left(\left|a_{n}\right|\right)$ converges to $\left.\log (\mid f(0)]\right)-$ $\log \|f\|$.

In Proposition 13, we shall use these classical lemmas 3,4,5,6 [8]:

Lemma 3: Let $a \in K$ and $r>0$ and $b \in d(a, r)$. Then $\varphi_{a, r}=\varphi_{b, r}$.

Lemma 4: Let $f \in A$. For every $r \in] 0,1[, f$ has finitely many zeroes. Let $a \in C(0, r)$. If $f$ has no zero in $d\left(a, r^{-}\right)$then $|f(x)|=|f|(r) \forall x \in d\left(a, r^{-}\right)$.

Moreover, the following 3 statements are equivalent:

i) $f$ is invertible in $A$.

ii) $f$ has no zeroes in $D$

iii) $|f(x)|$ is a constant in $D$.

Lemma 5: Let $f, g \in A$ be such that every zero a of $f$ is a zero of $g$ of order superior or equal to its order as a zero of $f$. Then there exists $h \in A$ such that $g=f h$. 
Lemma 6: Let $f \in A$. Then $\left|f^{\prime}\right|(r) \leq \frac{|f|(r)}{r} \forall r<1$.

By classical results on analytic functions we know this lemma (for instance [8] Th. 23.13):

Lemma 7: Let $f, \in A$ admits zeroes $a_{1}, \ldots a_{q}$ of respective order $k_{j}, j=1, \ldots, q$ in $\Gamma\left(0, r^{\prime}, r^{\prime \prime}\right)$. Then

$$
|f|\left(r^{\prime \prime}\right)=|f|\left(r^{\prime}\right) \prod_{j=1}^{q}\left(\frac{r^{\prime \prime}}{\left|a_{j}\right|}\right)^{k_{j}}
$$

In the proof of Theorem 3, we shall need the following lemma:

Lemma 8: Let $f(x)=\sum_{n=0}^{\infty} a_{n} x^{n} \in H(C(0, r))$ and assume that $f$ has a unique zero $\alpha$, of order 1 , in $C(0, r)$. Then $\left.\left|f^{\prime}\right|(\alpha) \mid\right)=\left|f^{\prime}\right|(r)$.

Proof: By hypothesis, $f(x)$ is of the form $(x-\alpha) h(x)$ with $h \in H(C(0, r))$, having no zero in $C(0, r)$. Then $|f|(r)=r|h|(r)$. Moreover, since $h$ has no zero in $C(0, r)$, we have $|h(\alpha)|=|h|(r)$. And by Lemma $6,\left|f^{\prime}\right|(r) \leq \frac{|f|(r)}{r}$. Therefore, we have $\left|f^{\prime}(\alpha)\right| \leq\left|f^{\prime}\right|(r) \leq$ $\frac{|f|(r)}{r}=|h|(r)=|h(\alpha)|=\left|f^{\prime}(\alpha)\right|$ and hence $\left|f^{\prime}(\alpha)\right|=\left|f^{\prime}\right|(r)$.

The following Theorem is given in [8] as Theorem 25.5.

Theorem F: Let $\left(a_{j}\right)_{j \in \mathbb{N}}$ be a sequence in $d\left(0,1^{-}\right)$such that $0<\left|a_{n}\right| \leq\left|a_{n+1}\right|$ for every $n \in \mathbb{N}$ and $\lim _{n \rightarrow \infty}\left|a_{n}\right|=r$. Let $\left(q_{n}\right)_{n \in \mathbb{N}}$ be a sequence in $\mathbb{N}^{*}$ and let $\left.B \in\right] 1,+\infty[$. There exists $f \in A\left(d\left(0, r^{-}\right)\right)$satisfying

i) $f(0)=1$

ii) $\|f\| \leq B \prod_{j=0}^{n}\left|\frac{a_{n}}{a_{j}}\right|^{q_{j}}$ whenever $n \in \mathbb{N}$

iii) for each $n \in \mathbb{N} a_{n}$ is a zero of $f$ of order $z_{n} \geq q_{n}$.

As a corollary of this Theorem, we can write Corollary F.1:

Corollary F.1: Let $\left(a_{j}\right)_{j \in \mathbb{N}}$ be a sequence in $d\left(0, r^{-}\right)$such that $0<\left|a_{n}\right| \leq\left|a_{n+1}\right|$ for every $n \in \mathbb{N}, \lim _{n \rightarrow \infty}\left|a_{n}\right|=r$ and let $\left(q_{n}\right)_{n \in \mathbb{N}}$ be a sequence in $\mathbb{N}^{*}$ such that

$$
\prod_{j=0}^{n}\left(\frac{\left|a_{n}\right|}{r}\right)^{q_{j}}>0 \text {. }
$$

Let $B \in] 1,+\infty[$. There exists $f \in A$ satisfying

i) $f(0)=1$

ii) $\|f\| \leq B \prod_{j=0}^{\infty}\left(\frac{r}{\left|a_{n}\right|}\right)^{q_{j}}$ whenever $n \in \mathbb{N}$

iii) for each $n \in \mathbb{N}, a_{n}$ is a zero of $f$ of order $z_{n} \geq q_{n}$. 
When $K$ is spherically complete, Lazard's Theorem is well known [16], [11]. Here we will just write it in the unit disk:

Theorem G: Let $K$ be spherically complete. Let $A^{\prime}$ be the $K$ algebra of all power series converging in D. Let $\left(a_{j}\right)_{j \in \mathbb{N}}$ be a coroner sequence and let $\left(q_{n}\right)$ be a sequence of positive integers. There exists $f \in A^{\prime}$ admitting each $a_{n}$ as a zero of order $q_{n}$ and having no other zeroes.

Consider a function $f \in A^{\prime}$ such that $f(0) \neq 0$, admitting for zeroes the $a_{n}$ with order 1 and no other zeroes. Then by Lemma 7 we have $|f|(r)=|f(0)| \prod_{\left|a_{j}\right|<r}\left(\frac{1}{\left|a_{j}\right|}\right)$. Consequently, such a function is bounded if and only if $\prod_{n=0}^{\infty}\left|a_{n}\right|>0$. Thus, we can derive Corollary G1:

Corollary G.1: Let $K$ be spherically complete. Let $\left(a_{j}\right)_{j \in \mathbb{N}}$ be a coroner sequence such that $\prod_{n=0}^{\infty}\left|a_{n}\right|>0$. There exists $f \in A$ admitting each $a_{n}$ as a zero of order 1 and having no other zeroes.

Proof of Theorem 2: By Theorem 23.15 [8] we know that there exist bounded sequences $\left(a_{n}\right)_{n \in \mathbb{N}}$ in $D$ such that the sequence $\left|\frac{a_{n}}{a_{n+1}}\right|$ is strictly increasing and then the function

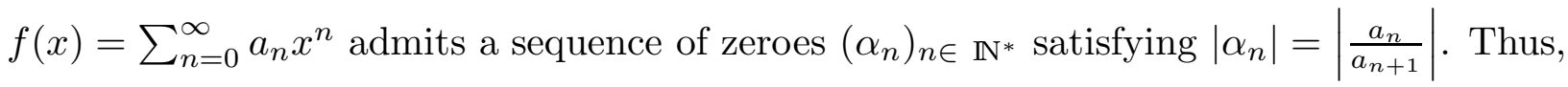
particularly, if we set $r_{n}=\left|\frac{a_{n}}{a_{n+1}}\right|$ then $f$ admits exactly a unique zero in each circle $C\left(0, r_{n}\right)$, each of order 1 , and has no other zero in $D$. Consequently, by Lemma 8 , we can see that $\left|f^{\prime}\left(\alpha_{n}\right)\right|=\left|f^{\prime}\right|\left(r_{n}\right) \forall n \in \mathbb{N}^{*}$. Now, let $\mathcal{U}$ be an ultrafilter thinner than the sequence $\left(\alpha_{n}\right)_{n \in \mathbb{N}^{*}}$. On the other hand, we can check that the sequence $\left(\alpha_{n}\right)$ is regular, hence $\mathcal{U}$ is a regular ultrafilter. Consequently, by Theorem $\mathrm{C}, \mathcal{J}(\mathcal{U})$ is a maximal ideal of $A$. Now, $f$ belongs to $\mathcal{J}(\mathcal{U})$ but $f^{\prime}$ doesn't.

Now, for each $n \in \mathbb{N}$, let $u_{n}=\log \left(r_{n}\right)$. Then, by Lemma 2, we have $\log (\|f\|)=$ $-\sum_{n=0}^{\infty} u_{n}$. By Lemma 1 , there exists an increasing sequence $\left(s_{n}\right)_{n \in \mathbb{N}}$ of $\mathbb{N}$ such that $\lim _{n \rightarrow \infty} s_{n}=+\infty$ and such that the series $\sum_{n=0}^{\infty} s_{n} u_{n}$ converges.

Now, by Corollary F1, there exists $g \in A$ (not identically zero) such that for each $n \in \mathbb{N}, \alpha_{n}$ is a zero of $g$ of order $z_{n} \geq s_{n}$. And since $\lim _{n \rightarrow \infty} s_{n}=+\infty$, for every fixed $k \in \mathbb{N}$, we can see that $f^{(k)}\left(\alpha_{n}\right)=0$ when $n$ is big enough, therefore $g^{(k)}$ belongs to $\mathcal{M}$. Consequently, $\widetilde{\mathcal{M}}$ is not null, which ends the proof.

Lemma 9: Let $\left(a_{n}\right)_{n \in \mathbb{N}}$ be a regular sequence, let $\delta=\inf _{k \in \mathbb{N}} \prod_{n \neq k, n \in \mathbb{N}}\left|a_{n}-a_{k}\right|$ and let $\rho=\inf _{k \neq n, k, n \in \mathbb{N}}\left|a_{n}-a_{k}\right|$. Let $f \in A$ admit each $a_{n}$ as a zero of order 1 and have no other zero. Then $\left|f^{\prime}(x)\right| \geq\|f\| \delta^{2} \forall x \in \bigcup_{n=0}^{\infty} d\left(a_{n},(\delta \rho)^{-}\right)$.

Proof: Let us fix $t \in \mathbb{N}$, let $r=\left|a_{t}\right|$ and let $E=d\left(a_{t}, r-\right)$. Set $u=x-a_{t}, g(u)=f(x)$ and consider $|g|(\delta)$. The zeroes of $g$ in $D \backslash d\left(0, \delta^{-}\right)$are the $a_{n}-a_{t}$ with $n \neq t$. Consequently, by Lemma 7, we can check that $|g|\left(\rho^{-}\right)=\|g\| \prod_{n \neq t}\left|a_{n}-a_{t}\right|$ and therefore 
$|g|(\rho) \geq\|g\| \delta=\|f\| \delta$.

Consider now $\left|g^{\prime}(u)\right|$ inside $d\left(0, \rho^{-}\right)$. Since $g$ has a unique zero at 0 in this disk, by Lemma 7 we have $|g|(r)=\left(\frac{r}{\rho}\right)|g|(\rho)$. Now, $g(u)$ is of the form $u h(u)$, with $h \in A$ having no zero in $d\left(0, \rho^{-}\right)$. Thus $h$ is of the form $c(1+l(u))$ with $l \in A$ and $|l(u)|<1 \forall u \in d\left(0, \rho^{-}\right)$, hence $|l|(r)<1 \forall r<\rho$. Now, $g^{\prime}(u)=c\left(1+l(u)+u l^{\prime}(u)\right)$. Now, in $d\left(0, \rho^{-}\right)$, since $|l(u)|(r)<1$, by Lemma 6 we have $\left|l^{\prime}(u)\right|(r)<\frac{1}{r}$ and hence $\left|u l^{\prime}(u)\right|(r)<1$. Consequently, $\left.\mid 1+l(u)+u l^{\prime}(u)\right) \mid=1 \forall u \in d\left(0, \rho^{-}\right)$and therefore

(2) $\left|g^{\prime}(u)\right|=|c| \forall u \in d\left(0, \rho^{-}\right)$.

Now, $|g|(\rho)=|u h(u)|(\rho)=\rho|h|(\rho)$. Since $|h(u)|$ is the constant $|c|$ inside $d\left(0, \rho^{-}\right)$, by (1) we can see that $|c| \geq \frac{\|f\| \delta}{\rho}$. Hence, by (2) we have $\left|g^{\prime}(u)\right| \geq \frac{\|f\| \delta}{\rho} \forall u \in d\left(0, \rho^{-}\right)$, i.e. $\left|f^{\prime}(x)\right| \geq \frac{\|f\| \delta}{\rho} \forall x \in d\left(a_{t}, \rho^{-}\right)$. This is true for every zero $a_{t}$ of $f$ and therefore, by setting $E=\bigcup_{n=0}^{\infty} d\left(a_{n}, \delta^{-}\right)$, we have $\left|f^{\prime}(x)\right| \geq \frac{\|f\| \delta}{\rho} \forall x \in E$. But clearly $E$ lies in $\mathcal{U}$ and therefore $f^{\prime}$ does not belong to $\mathcal{J}(\mathcal{U})$.

Proof of Theorem 3: Since $\mathcal{M}$ is a regular maximal ideal, there exists a regular sequence $\left(a_{n}\right)$ and a regular ultrafilter $\mathcal{U}$ thinner than the sequence $\left(a_{n}\right)$ such that $\mathcal{M}=$ $\mathcal{J}(\mathcal{U})$. Since the sequence is regular, we have $\delta=\inf _{k \in \mathbb{N}} \prod_{n \neq k, n \in \mathbb{N}}\left|a_{n}-a_{k}\right|>0$ and $\rho=\inf _{k \neq n, k, n \in \mathbb{N}}\left|a_{n}-a_{k}\right|>0$

Since $K$ is spherically complete, since $\delta>0$ we may apply Corollary G.1 showing there exists $f \in A$ admitting each $a_{n}$ as a zero of order 1 . Now by Lemma 9, we have $\left|f^{\prime}(x)\right| \geq\|f\| \delta^{2} \forall x \in \bigcup_{n=0}^{\infty} d\left(a_{n},(\delta \rho)^{-}\right)$which shows that $\varphi_{\mathcal{U}}\left(f^{\prime}\right)>0$. Consequently, $f^{\prime}$ does not belong to $\mathcal{M}$.

Lemma 10: Let $\psi \in \operatorname{Mult}(A, \|$. $\|)$ satisfy $\psi(P)=\|P\| \forall P \in K[x]$. Every quasiinvertible element $f \in A$ also satisfies $\psi(f)=\|f\|$.

Proof : First suppose $f \in$ invertible in $A$. Then $1=\psi(f) \psi\left(f^{-1}\right)$. But $\psi(f) \leq$ $\|f\|, \psi\left(f^{-1}\right) \leq\left\|f^{-1}\right\|$, hence both inequalities must be equalities. Now, let $f=P g \in A$ be quasi-invertible, with $P \in K[x]$ and $g \in A$, invertible in $A$. Then $\psi(f)=\psi(P) \psi(g)=$ $\|P\|\|g\|=\|P g\|=\|f\|$.

Proof of Theorem 4: The proof takes advantage of the proof of a theorem in [3]. Suppose the claim is wrong. Let $\psi \in \operatorname{Mult}(A, \|$. \|) be an absolute value on $A$ different from the Gauss norm $\|$. $\|$ on $A$. So, there exists a circular filter $\mathcal{F}$ on $D$, of diameter $r \leq 1$ such that $\psi(P)=\varphi_{\mathcal{F}}(P) \forall P \in K[x]$. But by Theorem A, we know that $r=1$ and hence, the restriction of $\psi$ to $K[x]$ is the Gauss norm. Now, since $\psi$ is not the Gauss norm on $A$, there exists $f \in A$ such that $\psi(f)<\|f\|$. Actually, without loss of generality, we can choose $f \in A$ such that $\psi(f)<1 \leq\|f\|$. Let $\rho=\psi(f)$. And, up a change of origin, we can also assume that $f(0) \neq 0$. By Lemma $10, f$ is not quasi-invertible, hence $f$ has a sequence of zeroes $\left(a_{n}\right)_{n \in \mathbb{N}}$ in $D$, with $\left|a_{n}\right| \leq\left|a_{n+1}\right|$. For each $n \in \mathbb{N}$, let $q_{n}$ be the 
multiplicity order of $a_{n}$. By Lemma 2 we know that $\sum_{n=0}^{\infty}-q_{n} \log \left|a_{n}\right|<+\infty$. Consequently, by Lemma 1 there exists a sequence $t_{n}$ of strictly positive integers satisfying

$$
\begin{aligned}
& t_{n} \leq t_{n+1}, n \in \mathbb{N}, \\
& \lim _{n \rightarrow \infty} t_{n}=+\infty, \\
& \sum_{n=0}^{\infty} t_{n} q_{n} \log \left(\left|a_{n}\right|\right)<+\infty .
\end{aligned}
$$

By Corollary $\mathrm{F} 1$ there exists a function $g \in A$ admitting each $a_{n}$ as a zero of order $s_{n} \geq t_{n} q_{n}$, such that $|g|\left(\left|a_{n}\right|\right) \leq 2\left|\prod_{k=0}^{n}\left(\frac{a_{n}}{a_{k}}\right)^{t_{n} q_{n}}\right| \forall n \in \mathbb{N}$ and consequently, $g$ belongs to $A$.

Now, for each $n \in \mathbb{N}$ and for each $k=0, \ldots, n$, let $u_{n, k}=\max \left(0, t_{n} q_{k}-s_{k}\right)$ and let $P_{n}(x)=\prod_{k=0}^{n}\left(x-a_{k}\right)^{u_{n, k}}$. Clearly, all coefficients of $P_{n}$ lie in $D$ except the leading coefficient that is 1 . Consequently, $\left\|P_{n}\right\|=1 \forall n \in \mathbb{N}$ and therefore

(1) $\left\|P_{n} g\right\|=\|g\|$.

On the other hand, since the sequence $t_{n}$ is increasing, we can check that for each fixed $n \in \mathbb{N}$, each zero $a_{k}$ of $f^{t_{n}}$ is a zero of $P_{n} g$ of order $\geq t_{k} q_{k}$. Consequently, by Lemma 6 in the ring $A$ we can write $P_{n} g$ in the form $f^{t_{n}} \sigma_{n}$, with $\sigma_{n} \in A$.

By (1), we have $\left\|\sigma_{n}\right\|\left\|f^{t_{n}}\right\|=\|g\|$ hence, since $\|f\| \geq 1$, we can see that $\left\|\sigma_{n}\right\| \leq\|g\|$. But now, since the restriction of $\psi$ to $K[x]$ is $\|$. $\|$, we have $\psi\left(P_{n}\right)=1$, hence $\psi\left(P_{n} g\right)=$ $\psi\left(P_{n}\right) \psi(g)=\psi(g)$ and therefore

(2) $\psi(g)=\psi\left(f^{t_{n}} \sigma_{n}\right)=\psi(f)^{t_{n}} \psi\left(\sigma_{n}\right) \leq \rho^{t_{n}}\|g\|$.

Relation (2) holds for every $n \in \mathbb{N}$ hence $\lim _{n \rightarrow+\infty} \rho^{t_{n}}\|g\|=0$. Consequently, $\psi(g)=0$, a contradiction. This ends the proof of Theorem 4.

In Propositions 14 and 15 we will denote by $|\cdot|_{\infty}$ the archimedean absolute value on $\mathbb{R}$.

Let $B$ be a commutative $K$-algebra with unity and let $f \in B$. We denote by $\operatorname{sp}(f)$ the set of the $\alpha \in K$ such that $f-\alpha$ is not invertible in $B$.

Proving Proposition 12 requires the knowledge of monotonous filters on an infraconnected set, particularly $T$-filters. Let $E$ be a closed bounded infraconnected subset of $K$ and let $\widetilde{E}$ be the smallest disk of the form $d(0, r)$ containing $E$. Let $a \in \widetilde{E}$ and $R \in \mathbb{R}_{+}^{*}$ be such that $\Gamma(a, r, R) \cap E \neq \emptyset$ whenever $r \in] 0, R[(\operatorname{resp} . \Gamma(a, R, r) \cap D \neq \emptyset$ whenever $r>R)$. We call an increasing (resp. a decreasing) filter of center a and diameter $R$, on $E$ the filter $\mathcal{F}$ on $E$ that admits for basis the family of sets $\Gamma(a, r, R) \cap E(\operatorname{resp} . \Gamma(a, R, r) \cap E)$. For every sequence $\left(r_{n}\right)_{n \in \mathbb{N}}$ such that $r_{n}<r_{n+1}\left(\right.$ resp. $\left.r_{n}>r_{n+1}\right)$ and $\lim _{n \rightarrow \infty} r_{n}=R$, it is seen that the sequence $\Gamma\left(a, r_{n}, R\right) \cap E\left(\operatorname{resp} . \Gamma\left(a, R, r_{n}\right) \cap E\right)$ is a basis of $\mathcal{F}$ and such a basis will be called a canonical basis . 
For convenience, in a field that is not spherically complete, we also call a decreasing filter with no center, of canonical basis $\left(D_{n}\right)_{n \in \mathbb{N}}$ and diameter $R>0$, on $E$ the circular filter on $E$ that admits for basis a sequence $\left(D_{n}\right)_{n} \in \mathbb{N}$ in the form $D_{n}=d\left(a_{n}, r_{n}\right) \cap D$ with $D_{n+1} \subset D_{n}, r_{n+1}<r_{n}, \lim _{n \rightarrow \infty} r_{n}=R$, and $\bigcap_{n \in \mathbb{N}} d\left(a_{n}, r_{n}\right)=\emptyset$.

Now, given a monotonous filter $\mathcal{F}$ on $E$, it is called a $T$-filter if the holes of $E$ satisfy certain arithmetical condition [5], [8] also defined by $T$-sequences [7], [18].

From the classical Krasner Mittag-Leffler Theorem, here we can state Proposition 11 $([8],[16])$ :

Proposition 11: Let $E$ be a set of the form $d(0, R) \backslash \bigcup_{i \in J} d\left(a_{i}, r_{i}^{-}\right)$) (where $J$ is a set of indices). Then any element $h \in H(E)$ has a unique decomposition of the form $\sum_{n=0}^{\infty} h_{n}$ whereas $h_{0} \in H(d(0, R))$ and for each $n \geq 1, h_{n} \in H\left(K \backslash d\left(a_{i_{n}}, r_{i_{n}}^{-}\right)\right)$and $\lim _{|x| \rightarrow+\infty} h_{n}(x)=0$, where the sequence of holes $\left(d\left(a_{i_{n}}, r_{i_{n}}^{-}\right)\right)_{n \in \mathbb{N}}$ of $E$ is linked to $h$ and defines its decomposition. Then $\|h\|_{E}=\max \left(\left\|h_{0}\right\|_{d(0, R)}, \sup _{n \geq 1}\left(\left\|h_{n}\right\|_{K \backslash\left(d\left(a_{i_{n}}, r_{i_{n}}^{-}\right)\right.}\right)\right.$. Further, $h_{0}$ is of the form $\sum_{j=0}^{\infty} a_{0, j} x^{j}$ with $\left\|h_{0}\right\|_{d(0, R)}=\sup _{j \geq 0}\left|a_{0, j}\right| R^{j}$ and for $n \geq 1, h_{n}$ is of the form $\sum_{j=1}^{\infty} a_{n, j}\left(x-a\left(r_{i_{n}}\right)\right)^{-j}$ with $\left\|h_{n}\right\|_{K \backslash\left(d\left(a_{i_{n}}, r_{i_{n}}^{-}\right)\right)}=\sup _{j \geq 1}\left|a_{n, j}\right|\left(r_{i_{n}}\right)^{-j}$.

Proposition 12: Let $(B,\|\|$.$) be a commutative ultrametric K$-Banach algebra with unity. Suppose there exist $f \in B, \phi, \psi \in \operatorname{Mult}(B,\|\|$.$) such that \psi(f)<\phi(f), s p(f) \cap$ $\Gamma(0, \psi(f), \phi(f))=\emptyset$ and there exists $\epsilon \in] 0, \phi(f)-\psi(f)\left[\right.$ satisfying further $\left\|(f-a)^{-1}\right\| \leq$ $M \forall a \in \Gamma(0, \psi(f), \phi(f)-\epsilon)$. Then there exists $\gamma \in B$ such that $\psi(\gamma)=1, \phi(\gamma)=0$.

Proof: Let $\|$. $\|_{s i}$ be the spectral norm defined on $B$ defined as $\|f\|_{s i}=\lim _{n \rightarrow \infty} \sqrt[n]{\left\|f^{n}\right\|}$.

Then we know that $\|f\|_{s i}=\sup \{\theta(f) \mid \theta \in \operatorname{Mult}(B,\|\|)$.$\} .$

Let $s=\psi(f), t=\phi(f)-\epsilon, R=\|f\|$. We first check

(1) $M \geq \frac{1}{s}$.

Let $a \in \Gamma(0, s, t)$. Since $\psi(f)<|a|$, we have $\psi(f-a)=|a|$ hence $\psi\left((f-a)^{-1}\right)=\frac{1}{|a|}>\frac{1}{s}$ and therefore $\left\|(f-a)^{-1}\right\|_{s i} \geq \frac{1}{s}$. But of course $\|g\|_{s i} \leq\|g\| \forall g \in B$, so (1) is clear.

Now, let $L=\frac{1}{M}$. For each $\left.r \in|K| \cap\right] s, t$, we choose $a(r) \in C(0, r)$ and denote by $E$ the set $d(0, R) \backslash\left(\bigcup_{r \in] s, t[} d\left(a(r), L^{-}\right)\right)$. We notice that $d\left(a(r), L^{-}\right) \subset C(0, r)$. Now, there 
exists a natural homomorphism $\sigma$ from $H(E)$ into $B$ such that $\sigma(x)=f$. And since $R=\|f\|$ and $\left\|(f-b)^{-1}\right\| \leq M \forall b \in \Gamma(0, s, t)$, the topological properties of the KrasnerMittag-Leffler Theorem recalled above show that $\sigma$ is clearly continuous with respect to the norms $\|.\|_{E}$ of $H(E)$ and $\|$. $\|$ of $B$. Now, let $\psi^{\prime}=\psi \circ \sigma, \phi^{\prime}=\phi \circ \sigma$. Then both $\phi^{\prime}, \psi^{\prime}$ belong to $\operatorname{Mult}(H(E),\|\|$.$) and satisfy \psi^{\prime}(x)=s, \phi^{\prime}(x)=t$. So, $\psi^{\prime}$ is of the form $\varphi_{\mathcal{F}}$ with $\mathcal{F}$ a circular filter secant with $d(0, s)$ and $\phi^{\prime}$ is of the form $\varphi_{\mathcal{G}}$ with $\mathcal{G}$ a circular filter secant with $d(0, R) \backslash d\left(0, t^{-}\right)$.

By properties of $T$-filters, [8], [9], [18] we know that for every $r \in] s, t[, E$ admits an increasing idempotent $T$-sequence $d\left(a_{n}, L^{-}\right)$of center 0 and diameter $r$, such that $\left|a_{n}\right|<\left|a_{n+1}\right|$. Consequently, $E$ admits an increasing $T$-filter of center 0 and diameter $r$ (and similarly it admits a decreasing $T$-filter of center 0 and diameter $r$ ) [18]. Then by Theorem $37.2[8]$, there exists $h \in H(E)$ meromorphic on each hole $d\left(a_{n}, L^{-}\right)$such that $h(u)=0 \forall u \in d(0, R) \backslash d\left(0, r^{-}\right)$, admitting further each $a_{n}$ as a simple pole or a holomorphic point and no other pole in $d(0, R)$ [8]. Moreover, since $h$ is a quasi-invertible element in $H(d(0, s))$, we may choose $h$ having no zero in $d(0, s)$. Consequently, $|h(x)|$ is then constant in $d(0, s)$ and may be taken equal to 1 .

Let $\gamma=\sigma(h)$. Then $\psi(\gamma)=\psi^{\prime}(h)=1$ and $\phi(\gamma)=\phi^{\prime}(h)=0$, which ends the proof.

By Theorem (3.2) in [19], we have the following Proposition 13:

Proposition 13: Let $f_{1}, \ldots, f_{q} \in A$ satisfy $\left\|f_{j}\right\|<1 \forall j=1, \ldots, q$ and $\inf \left\{\max _{j=1, \ldots, q}\left(\left|f_{j}(x)\right|\right) \mid x \in D\right\}=\omega>0$. There exist $g_{1}, \ldots, g_{q} \in A$ such that $\sum_{j=1}^{q} g_{j} f_{j}=1$ and $\max _{j=1, \ldots, q}\left\|g_{j}\right\|<\omega^{-2}$.

Proposition 14: Let $\mathcal{M}$ be a non-principal maximal ideal of $A$ and let $\mathcal{U}$ be an ultrafilter on $D$ such that $\mathcal{M}=\mathcal{J}(\mathcal{U})$. Let $f \in A \backslash \mathcal{M}$ be not invertible in $A$ and let $g \in A, h \in \mathcal{M}$ such that $f g=1+h$. Let $\lambda=\varphi_{\mathcal{U}}(f)$, let $\left.\epsilon \in\right] 0, \min (\lambda, 1)[$ and let $\Lambda=\left\{x \in D|| f(x) g(x)|-1|_{\infty}<\epsilon,|| f(x)|-\lambda|_{\infty}<\epsilon\right\}$.

Suppose that there exist a function $\tilde{h} \in A$ admitting for zeroes in $D$ the zeroes of $h$ in $D \backslash \Lambda$ and a function $\widehat{h} \in A$ admitting for zeroes the zeroes of $h$ in $\Lambda$, each counting multiplicities, so that $h=\widetilde{h} \widehat{h}$. Then $|\widetilde{h}(x)|$ has a strictly positive lower bound in $\Lambda$ and $\widehat{h}$ belongs to $\mathcal{M}$.

Moreover, there exists $\omega \in] 0, \lambda[$ such that $\omega \leq \inf \{\max (|f(x)|,|\widehat{h}(x)|) \mid x \in D\}$. Further, for every $a \in d(0,(\lambda-\epsilon))$, we have $\omega \leq \inf \{\max (|f(x)-a|,|\widehat{h}(x)|) \mid x \in D\}$.

Proof: Let $u \in \Lambda$ and let $s$ be the distance of $u$ from $K \backslash \Lambda$. So, the disk $d\left(u, s^{-}\right)$is included in $\Lambda$, hence $f g$ has no zero inside this disk. Consequently, $|f(x) g(x)|$ is a constant $b$ in $d\left(u, s^{-}\right)$. Consider the family $F_{u}$ of radii of circles $C(u, r)$, containing at least one zero of $f g$. By Lemma $4 F_{u}$ has no cluster point different from 1. Consequently, there exists $\rho \geq s$ such that $f g$ admits at least one zero in $C(u, \rho)$ and admits no zero in $d\left(u, \rho^{-}\right)$. And 
then $|f(x) g(x)|$ is a constant $c$ in $d\left(u, \rho^{-}\right)$. But then, at $u$ we see that $b=c$ and therefore $d\left(u, \rho^{-}\right)$is included in $\Lambda$. Hence $\rho=s$ and therefore $f g$ admits at least one zero $\alpha$ in $C(u, s)$. Thus, at $\alpha$ we have $h(\alpha)=-1$. Therefore, in the disk $d\left(\alpha, s^{-}\right)$we can check that $\varphi_{\alpha, s}(h) \geq 1$. But by Lemma $3, \varphi_{\alpha, s}(h)=\varphi_{u, s}(h)$, hence $\varphi_{u, s}(h) \geq 1$.

Now,

$$
\frac{\|h\|}{\varphi_{u, s}(h)}=\frac{\|\widetilde{h}\|}{\varphi_{u, s}(\widetilde{h})} \frac{\|\widehat{h}\|}{\varphi_{u, s}(\widehat{h})} \geq \frac{\|\widetilde{h}\|}{\varphi_{u, s}(\widetilde{h})} .
$$

Therefore, since $\varphi_{u, s}(h) \geq 1$, we obtain

$$
\frac{\|\widetilde{h}\|}{\varphi_{u, s}(\widetilde{h})} \leq\|h\|
$$

But since by definition $d\left(u, s^{-}\right)$is included in $\Lambda, \widetilde{h}$ has no zero in this disk, hence $|\widetilde{h}(x)|$ is constant and equal to $\varphi_{u, s}(\widetilde{h})$. Consequently, by (1) we obtain $\frac{\|\widetilde{h}\|}{|\widetilde{h}(u)|} \leq\|h\|$ and therefore we have

$$
|\widetilde{h}(u)| \geq \frac{\|\widetilde{h}\|}{\|h\|} \forall u \in \Lambda \text {. }
$$

This shows that $\widetilde{h}$ does not belong to $\mathcal{M}$. Consequently, $\widehat{h}$ does belong to $\mathcal{M}$.

Now, by hypothesis, we have $f g-\widehat{h} \widetilde{h}=1$. Since both $g, \widetilde{h}$ belong to $A$ and therefore are bounded in $D$, it is obvious that $\inf \{\max (|f(x)|,|\widehat{h}(x)|) \mid x \in D\}>0$. So, we may obviously choose $\omega \in] 0, \lambda-\epsilon[\operatorname{such}$ that $\omega \leq \inf \{\max (|f(x)|,|\widehat{h}(x)|) \mid x \in D\}$.

Let $\Lambda^{\prime}=\{x \in D|| f(x) \mid \geq \lambda-\epsilon\}$ and let $a \in d\left(0,(\lambda-\epsilon)^{-}\right)$. When $\beta$ lies in $\Lambda^{\prime}$, we have $|f(\beta)|>|a|$, hence $\max (|f(\beta)-a|,|\widehat{h}(\beta)|) \geq \omega$. Now, let $\beta$ lie in $D \backslash \Lambda^{\prime}$ and let $\tau$ be the distance from $\beta$ to $\Lambda^{\prime}$. Since $D \backslash \Lambda^{\prime}$ is open, $\tau$ is $>0$. Thus, we have $\varphi_{\beta, \tau}(f) \geq \lambda-\epsilon$. If $f$ had no zero in $d\left(\beta, \tau^{-}\right)$, we would have $\varphi_{\beta, \tau}(f)=|f(\beta)|<\lambda-\epsilon$, a contradiction. Hence $f$ must have a zero $\gamma$ in $d\left(\beta, \tau^{-}\right)$. Then $|\widehat{h}(\gamma)| \geq \omega$. But since by definition, $\Lambda \subset \Lambda^{\prime}$, the zeroes of $\widehat{h}$ belong to $\Lambda^{\prime}$. And since $d\left(\beta, \tau^{-}\right) \cap \Lambda^{\prime}=\emptyset$ actually $\widehat{h}$ has no zero in $d\left(\beta, \tau^{-}\right)$. Consequently $|\widehat{h}(x)|$ is constant in $d\left(\beta, \tau^{-}\right)$and hence $|\widehat{h}(\beta)| \geq \omega$, which completes the proof.

By Propositions 11, 12, 13 we can easily state Proposition 15:

Proposition 15: Suppose $K$ is spherically complete. Let $\mathcal{M}$ be a non-principal maximal ideal of $A$ and let $\mathcal{U}$ be an ultrafilter on $D$ such that $\mathcal{M}=\mathcal{J}(\mathcal{U})$. Let $f \in A \backslash \mathcal{M}$ satisfy $\|f\|<1$, let $\lambda=\varphi_{\mathcal{U}}(f)$ and let $\left.\epsilon \in\right] 0, \lambda[$. There exists $\omega>0$ such that, for every $a \in$ $d(0, \lambda-\epsilon)$, there exists $g_{a} \in A$ satisfying $(f-a) g_{a}-1 \in \mathcal{M}$ and $\left\|g_{a}\right\| \leq \omega^{-2}$.

Proof: Suppose first that $f$ is invertible in $A$, then by Lemma $4|f(x)|$ is a constant and hence is equal to $\lambda$. Therefore, $|f(x)-a|=\lambda \forall a \in d(0, \lambda-\epsilon)$. Consequently, $f-a$ is 
invertible and its inverse $g_{a}$ satisfies $\left\|g_{a}\right\|=\lambda^{-1}$. Thus, we only have to show the claim when $f$ is not invertible.

Since $f$ does not belong to $\mathcal{M}$, we can find $g \in A$ and $h \in \mathcal{M}$ such that $f g=1+h$. Let $\Lambda=\left\{x \in D|||f(x) g(x)|-\left.1\right|_{\infty}<\epsilon,|| f(x)|-\lambda|_{\infty}<\epsilon\right\}$.

Since $K$ is spherically complete, we can factorize $h$ in the form $\widetilde{h} \widehat{h}$ where $\widetilde{h} \in A$ is a function admitting for zeroes in $D$ the zeroes of $h$ in $D \backslash \Lambda$ and $\widehat{h} \in A$ is a function admitting for zeroes the zeroes of $h$ in $\Lambda$, each counting multiplicities, so that $h=\widetilde{h} \widehat{h}$. Moreover, we can choose $\widetilde{h} \widehat{h}$ so that $\|\widehat{h}\|<1$. Thus, we have $f g-(\widehat{h})(\widetilde{h})=1$, with $\|f\|<1,\|\widehat{h}\|<1$. By Proposition 14, there exists $\omega>0$ such that $\omega \leq \inf \{\max (|f(x)|,|\widehat{h}(x)|) \mid x \in D\}$ and that for every $a \in d(0,(\lambda-\epsilon))$, we have $\omega \leq \inf \{\max (|f(x)-a|,|\widehat{h}(x)|) \mid x \in D\}$. Now, we notice that $\|f-a\|<1$ for every $a \in d(0, \lambda-\epsilon)$, so we may apply Proposition 13 and obtain $g_{a}, h_{a} \in A$ such that $(f-a) g_{a}+\widehat{h} h_{a}=1$, with $\left\|g_{a}\right\|<\omega^{-2},\left\|h_{a}\right\|<\omega^{-2}$. Since $\widehat{h} h_{a}$ belongs to $\mathcal{M},(f-a) g_{a}-1$ belongs to $\mathcal{M}$, which completes the proof.

Proof of Theorem 5: Suppose $A$ is not multbijective and let $\mathcal{M}$ be a maximal ideal which is not univalent. Let $F$ be the quotient field $\frac{A}{\mathcal{M}}$, let $\theta$ be the canonical surjection from $A$ onto $F$ and let $\|$. $\|_{q}$ be the $K$-Banach algebra quotient norm of $F$. By [10] (Corollary 12.1) there exists an ultrafilter $\mathcal{U}$ on $D$ such that $\mathcal{M}=\mathcal{J}(\mathcal{U})$. Thus, there exists $\psi \in \operatorname{Mult}(A, \|$. $\|)$ such that $\operatorname{Ker}(\psi)=\mathcal{M}$ and $\psi \neq \varphi \mathcal{U}$. Consequently, there exists $f \in A$ such that $\psi(f) \neq \varphi_{\mathcal{U}}(f)$, with $\psi(f) \neq 0, \varphi_{\mathcal{U}}(f) \neq 0$. We shall check that we may also assume $\psi(f)<\varphi_{\mathcal{U}}(f)$. Indeed, suppose $\psi(f)>\varphi_{\mathcal{U}}(f)$. Let $g \in A$ be such that $\theta(g)=\theta(f)^{-1}$. Then we can see that $\psi(g)=\psi(f)^{-1}, \varphi_{\mathcal{U}}(g)=\left(\varphi_{\mathcal{U}}(f)\right)^{-1}$, therefore $\psi(g)<\varphi_{\mathcal{U}}(g)$. Thus, we may assume $\psi(f)<\varphi_{\mathcal{U}}(f)$ without loss of generality. Similarly, we may obviously assume that $\|f\|<1$. By Lemma 10, we know that $f$ is not invertible.

Let $\lambda=\varphi_{\mathcal{U}}(f)$ and let $\left.\epsilon \in\right] 0, \lambda[$. By Proposition 15, there exists $\omega>0$ such that, for every $a \in d(0, \lambda-\epsilon)$, there exists $g_{a} \in A$ satisfying $(f-a) g_{a}-1 \in \mathcal{M}$ and $\left\|g_{a}\right\| \leq \omega^{-2}$. Now, $\theta\left(g_{a}\right)=(\theta(f-a))^{-1}$. Thus, $\left\|(\theta(f-a))^{-1}\right\|_{q} \leq \omega^{-2} \forall a \in d(0, \lambda-\epsilon)$. Now, by applying Proposition 12 to the $K$-Banach algebra $F$, we can see that there exists $y \in F$ such that $\varphi_{\mathcal{U}}(y)=0, \psi(y)=1$, a contradiction to the hypothesis $\operatorname{Ker}\left(\varphi_{\mathcal{U}}\right)=\operatorname{Ker}(\psi)$. This finishes showing that $A$ is multbijective.

\section{References}

[1] Berkovich, V. Spectral Theory and Analytic Geometry over Non-archimedean Fields. AMS Surveys and Monographs 33, (1990).

[2] Boussaf, K. and Escassut, A. Absolute values on algebras of analytic elements Annales Mathématiques Blaise Pascal 2, n2, p.15-23 (1995).

[3] Boussaf, K. Identity theorem for bounded meromorphic P-adic functions Preprint.

[4] Carleson, L. Interpolation by bounded analytic functions and the corona problem Annals of Math. 76, p. 547-559 (1962). 
[5] Escassut, A. T-filtres, ensembles analytiques et transformations de Fourier padique. Annales de l'Institut Fourier, tome 25, fasc 2, pp 35-80 (1975).

[6] Escassut, A. Spectre maximal d'une algèbre de Krasner, Colloquium Mathematicum, XXXVIII, fasc. 2, p. 339-357, (1978).

[7] Escassut, A. The ultrametric spectral theory, Periodica Mathematica Hungarica, Vol.11, (1), p7-60, (1980).

[8] Escassut, A. Analytic Elements in p-adic Analysis, World Scientific Publishing Inc., Singapore (1995).

[9] Escassut, A. Ultrametric Banach Algebras, World Scientific Publishing Inc., Singapore (2003).

[10] Escassut, A. and Mainetti, N About the ultrametric Corona problem Bulletin des Sciences Mathématiques 132, p. 382-394 (2008)

[11] Escassut, A. p-adic Distribution Value Topics on value distribution and differentiability ...(Escassut, A., Yang, C.C., Wong, P.) Scientific Press, (2008)

[12] Garandel, G. Les semi-normes multiplicatives sur les algèbres d'éléments analytiques au sens de Krasner, Indag. Math., 37, n4, p.327-341, (1975).

[13] Guennebaud, B. Sur une notion de spectre pour les algèbres normées ultramétriques, thèse Université de Poitiers, (1973).

[14] Hoffman, K. Banach Spaces of Analytic Functions. Prentice-Hall Inc. (1962).

[15] Krasner, M. Prolongement analytique uniforme et multiforme dans les corps valués complets. Les tendances géométriques en algèbre et théorie des nombres, ClermontFerrand, p.94-141 (1964). Centre National de la Recherche Scientifique (1966), (Colloques internationaux du C.N.R.S. Paris, 143).

[16] Lazard, M. Les zéros des fonctions analytiques sur un corps valué complet, IHES, Publications Mathématiques n14, p.47-75 ( 1962).

[17] ROBERT, A. A Course in p-adic Analysis . Vol.198, 2000, Springer

[18] Sarmant, M.-C. and Escassut, A. T-suites idempotentes, Bull. Sci. Math. 106, p.289-303, (1982).

[19] Van Der Put, M. The Non-Archimedean Corona Problem Table Ronde Anal. non Archimedienne, Bull. Soc. Math. Mémoire 39-40, p. 287-317 (1974).

Alain Escassut

Laboratoire de Mathématiques UMR 6620

Université Blaise Pascal

(Clermont-Ferrand)

Les Cézeaux

63177 AUBIERE CEDEX

FRANCE

Alain.Escassut@math.univ-bpclermont.fr 